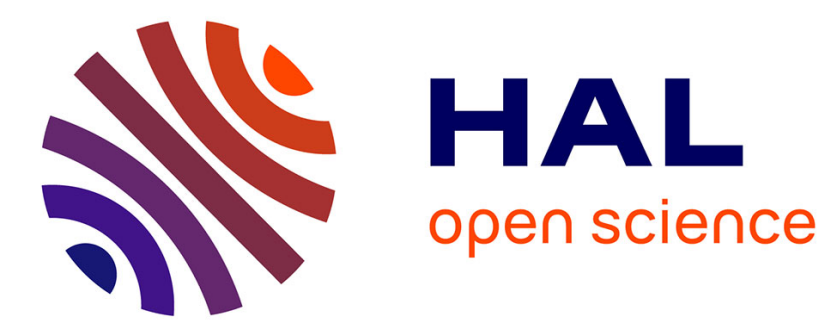

\title{
CONTROLLING TESTING MACHINES WITH DIGITAL IMAGE CORRELATION
}

\author{
Xavier Fayolle, Sylvain Calloch, François Hild
}

\section{To cite this version:}

Xavier Fayolle, Sylvain Calloch, François Hild. CONTROLLING TESTING MACHINES WITH DIGITAL IMAGE CORRELATION. Experimental Techniques, 2007, 31 (3), pp.57-63. 10.1111/j.17471567.2007.00152.x . hal-00199383

\section{HAL Id: hal-00199383 https://hal.science/hal-00199383}

Submitted on 18 Dec 2007

HAL is a multi-disciplinary open access archive for the deposit and dissemination of scientific research documents, whether they are published or not. The documents may come from teaching and research institutions in France or abroad, or from public or private research centers.
L'archive ouverte pluridisciplinaire HAL, est destinée au dépôt et à la diffusion de documents scientifiques de niveau recherche, publiés ou non, émanant des établissements d'enseignement et de recherche français ou étrangers, des laboratoires publics ou privés. 
Submitted to Exp. Tech., May 2005

Revised, August 2006

\section{Controlling testing machines with digital image correlation}

Xavier FAYOLLE, Sylvain CALLOCH and François HILD ${ }^{1}$

LMT-Cachan

ENS Cachan / CNRS-UMR 8535 / Université Paris 6

61 avenue du Président Wilson, F-94235 Cachan Cedex, France

${ }^{1}$ Corresponding author. Fax: +331474022 40. Email: hild@Imt.ens-cachan.fr 


\title{
Controlling testing machines with digital image correlation
}

by

Xavier FAYOLLE, Sylvain CALLOCH and François HILD

\begin{abstract}
It is proposed to discuss a strategy to control a mechanical test by using digital image correlation. A two-loop system is developed to integrate a sub-pixel correlation algorithm to evaluate the average strain of a region of interest and control a servo-hydraulic testing machine. An a priori uncertainty analysis is validated a posteriori with actual strain measurements. A tensile test was successfully carried out with the developed system.
\end{abstract}




\section{Introduction}

In the past, tensile / compressive tests that give the elementary mechanical properties of materials such as Young's Modulus, Poisson's ratio, yield stress and ultimate strength were common practice. Nowadays, with the low cost and easy to get computation means, the behavior of structures is simulated by using non-linear constitutive equations. These computation codes need material-dependent parameters to described different types of behaviors. Consequently, there is a demand for complex mechanical tests on materials. During the last decade, tests have become increasingly complicated, for instance, by inducing heterogeneous strains, or by including thermomechanical load histories. To analyze them, full-field measurement techniques are developed and implemented. ${ }^{1,2}$

When the test is strain-controlled, one may use strain gauges or extensometers. Digital image correlation (DIC) is one of the optical solutions ${ }^{2}$ that can be used to control a test. This is achieved, for instance, with a camera taking pictures that are processed subsequently to give full-field displacement fields of sample surfaces. The typical standard displacement uncertainty $\rho_{u}$ is less than $10^{-2}$ pixel for 8 -bit-coded pictures. ${ }^{3}$ Depending on the gauge length $L_{0}$, the corresponding standard strain uncertainty $\rho_{\varepsilon}$ is of the order of $\rho_{u} / L_{0}$ (i.e., $10^{-4}$ when $L_{0}=100$ pixels, or $10^{-5}$ when $L_{0}=1000$ pixels).

The aim of the paper is to show the feasibility of controlling strains in a mechanical test by using digital image correlation. A software, called CORRELI ${ }^{\mathrm{CONTROL}}$, was developed and is presented herein. It is validated by driving a tensile test on a steel sample. Section 2 is devoted to the description and performance of the correlation algorithm used in CORRELI ${ }^{\text {CONTROL }}$. In particular, the correlation parameters are chosen and the strain uncertainty is determined a priori. Cascade feedback architecture is introduced, tuned and characterized in Section 3. In Section 4, a tensile test on steel is controlled via CORRELI ${ }^{\text {CONTROL }}$. 


\section{Correlation algorithm and strain uncertainty}

DIC will be used to control an experiment. In the present case, a strain history is prescribed. Consequently, to design the controller, the performance of the DIC algorithm has to be evaluated (i.e., the strain uncertainty associated with the correlation parameters, namely, the correlation window size and the separation between two consecutive windows). The strain uncertainty depends upon the considered picture and the correlation algorithm itself. The latter is first presented and the former is then determined.

In practice, one starts by selecting the region of interest (ROI) for the optical gauge. Within the ROI, smaller correlation windows or zones of interest (ZOIs) are chosen. For each $\mathrm{ZOI}$ in the reference image, one determines its corresponding position in subsequent pictures. The pattern-matching algorithm is based on cross-correlation. The cross-correlation is a function giving the maximum likelihood between two ZOIs shifted by a constant displacement. Its computation is performed via fast Fourier transforms. The location of this maximum gives the most likely displacement between the reference ZOI and the subsequent one. For a sub-pixel estimate, a parabolic interpolation is performed around the maximum pixel value and the displacement corresponds to the maximum of interpolated correlation function. Then the reference ZOI is shifted by an amount equal to the evaluated displacement using the shift/modulation property. One re-iterates these three steps until convergence is reached. In practice, three iterations are sufficient to achieve corrections less than $5 \times 10^{-3}$ pixel (i.e., the standard displacement uncertainty). The interested reader will find additional details given by Périé et al. ${ }^{3}$ and Hild et $a .^{4}$ In the present case, since very small strain and displacement increments are sought, a constant displacement hypothesis for each ZOI is deemed to be sufficient.

To devise the feedback control loop using DIC, an estimate of the strain uncertainty of the correlation algorithm is needed. The following procedure is applied to the actual picture 
of the experiment described in Section 4. The CCD digital camera used herein has a $1280 \times 1024$-pixel resolution and a 12-bit dynamic range. If careful attention is paid to the product specifications, one notes that the least significant bits of the analog to digital converter is corrupted by readout noise so that the sensor's actual dynamic range is estimated to be 10.5 bits. $^{5}$ To have independent estimates of the displacements in the following analyses, the shift $\delta$ between consecutive ZOIs is equal to the ZOI size $L_{Z O I}$.

First, the standard displacement uncertainty ${ }^{6}$ is estimated. A constant displacement equal to $1 / 2$ pixel along both directions is applied artificially by using the shift/modulation property of Fourier transforms. ${ }^{3}$ The average difference gives an evaluation of the error and the standard deviation that of the corresponding uncertainty. With the present correlation algorithm, a half-pixel displacement corresponds to the worst case and the standard uncertainty is one half of the corresponding standard deviation. ${ }^{7}$ Figure 1 shows the standard uncertainty as a function of the ZOI size; the larger the ZOI size, the smaller the uncertainty. However, if the ZOI size increases, the spatial resolution is degraded, i.e., less independent measurement points are obtained (i.e., $\left\lfloor L_{R O I x} / L_{Z O I}\right\rfloor \times\left\lfloor L_{R O I y} / L_{Z O I}\right\rfloor$, where $L_{R O I x}$ is the horizontal length of the ROI and $L_{\text {ROIy }}$ the vertical length, Fig. 6). For ZOI sizes greater than or equal to 16 pixels, a power law with an exponent of -1.5 is obtained for the standard uncertainty as a function of the ZOI size

$$
\rho_{u}=\frac{A^{1+\alpha}}{L_{Z O I}^{\alpha}},
$$

with $A=1.43$ pixels (and $\alpha=1.5$ ), thereby indicating that for a large range of ZOI sizes, the standard uncertainty and the corresponding spatial resolution are the result of a compromise described by Eqn. (1). The ZOI size also needs to be adapted to the speckle size (here of the order of 2 pixels when characterized by an average correlation radius, i.e., sufficiently small with respect to $\left.L_{Z O I}\right)$. Furthermore, as the ZOI size increases, so does the computation time. 
A good compromise for the present study is given by a ZOI size of 64 pixels for which a standard displacement uncertainty of $5 \times 10^{-3}$ pixel is achieved.

Second, the strain uncertainty is determined by using the same picture and the same displacement field. From the displacement field, the strains are evaluated by using a centered finite differences scheme so that the spatial resolution associated with strain evaluations (i.e., the gauge length) is equal to twice the shift between neighboring ZOIs. By dimensional analysis, the standard strain uncertainty is inversely proportional to the gauge length $L_{0}$ so that

$$
\rho_{\varepsilon}=B \frac{\rho_{u}}{L_{0}}
$$

with $B=1.3$, so that an increase of the gauge length yields a lower strain uncertainty, yet a poorer spatial resolution. If one assumes a normal distribution of displacement errors, $B=\sqrt{2} \approx 1.4,{ }^{8,9}$ which is close to value found above. Equation (2) is in agreement with the results shown in Fig. 1 since a power law with an exponent -2.5 is found when the gauge length is equal to two times the ZOI size. The origin of such dependence is understood by the fact that the centered finite difference operation relates the standard displacement uncertainty $\rho_{u}$ and that of the strain $\rho_{\varepsilon}$ as proportional to each other, and inversely proportional to the gauge length (here equal to $2 L_{\mathrm{ZOI}}$ ).

For strains, two parameters play a role in the uncertainty, namely, the ZOI size $L_{Z O I}$ and the gauge length $L_{0}$. The larger the ZOI size, the smaller $\rho_{\varepsilon}$, and the longer the computation time. For a fixed ZOI size, the larger the gauge length, the lower the strain uncertainty. In the present case, this property will be used since $L_{0}$ is chosen to be greater than $2 L_{Z O I}$ (as was used in the present analysis). Furthermore, an additional gain is obtained by considering more than two neighboring points to evaluate the average strain. When $3 \times 3$ measurements are considered with an identical separation between two neighboring points of $L_{0} / 2$, the constant $B$ in Eqn. (2) decreases, i.e., $B=\sqrt{2 / 3} \approx 0.8 .^{8}$ In terms of strains, the 
corresponding standard uncertainty is equal to $10^{-5}$ with a minimum gauge length $L_{0}$ of 400 pixels. In the present case, the ROI resolution is $550 \times 1000$ pixels to observe most of the central part of the sample (see Fig. 6). Consequently, the a priori strain uncertainty is of the order of $10^{-5}$ when $L_{Z O I}=64$ pixels and $L_{0}=400$ pixels.

\section{Feedback design, tuning and characterization}

To control an experiment with a given strain history, two routes can be followed. The first and easiest is to use the strain conditioning system directly in the hardware feedback loop of the testing machine ${ }^{10}$ (e.g., with strain gauges or extensometers). The sampling rate associated with the strain measurement has to be greater than the machine bandwidth. When the latter requirement is not satisfied, a cascade feedback has to be implemented, whereby the machine is controlled by a fast-reacting displacement-controlled inner loop with a slower outer strain-controlled loop, using a DIC algorithm.

Control theory applied to sampled systems states that if the sampling rate is over thirty times greater than the system bandwidth, then the rules of analog control theory is applicable as a good approximation. This theory also indicates that for systems where the sampling rate obeys inequations (3), one must work with Z-transforms to model the system ${ }^{11}$

$$
5 f_{b w}<f_{s}<25 f_{b w},
$$

where $f_{s}$ and $f_{b w}$ are the sampling rate and closed-loop system bandwidth, respectively. For sampling rates less than $3 f_{b w}$, any attempt to control the system becomes problematic, since fast transients may be missed. Besides, controlling a system in a poorly sampled way leads to important drifts. It will be shown that the computation time to acquire pictures, to store them, to measure the displacements, and to compute the average strain is around $6 \mathrm{~s}$ (this time may be optimized by using other programming languages). Therefore the sampling rate is at best equal to $0.15 \mathrm{~Hz}$. According to inequations (3), the system to control, i.e., the testing machine, needs a bandwidth narrower than $0.03 \mathrm{~Hz}$. This is not the case since bandwidths 
around $30 \mathrm{~Hz}$ are commonly implemented in electro-hydraulic testing machines. Consequently, cascade feedback architecture is chosen (Fig. 2a).

The aim of the following discussion is to show how a robust and self-controlled system was designed to drive a servo-hydraulic testing machine. One of the main points is to avoid any fatal troubleshooting (i.e., one does not want sample failure caused by an erratic feedback). This security step is achieved by limiting the command signal increment. This leads to a non-linear control scheme. A stable response together with a zero steady-state error is also sought. Figure 2 gives functional and block diagrams of the cascade control scheme selected. The strain-controlled loop includes the inner reactive loop, which is displacementcontrolled in the present case. A PC is used for image processing and the outer loop controller. The whole signal processing and control software were developed in LabVIEW environment. $^{12}$

The outer loop is driven in a static way, i.e., the reference strain signal is set step-wise and, in-between two steps, the machine (inner loop) response is let to completely settle down (i.e., with a steady state error less than, say, the strain uncertainty). Furthermore, nonlinearity is implemented by limiting (clipping) the controller output command increment. One thus sets the reference strain signal of the outer loop as a series of steps with constant and small increments.

The different parameters of the outer loop need to be tuned. To calculate the gain $K_{\mathrm{i}}$ of the integral term of the controller (Fig. 2b), an estimate of the open loop gain is first sought, which depends on the strain uncertainty of the DIC technique. The error limit is chosen equal to $2 \times 10^{-5}$, i.e., twice the standard strain uncertainty with the chosen correlation parameters (Section 2). The corresponding displacement increment is such that $\delta U=2 \rho_{\varepsilon} L_{g}$, where $L_{g}$ is the sample gauge length. The computer data acquisition board has two 16-bit digital to analog converters (DAC) with a $\pm 10 \mathrm{~V}$ working range. The DAC resolution is 
therefore equal to $0.3 \mathrm{mV}$ (i.e., $20 / 2^{16} \mathrm{~V}$ ). One takes one DAC increment for $\delta U$. Therefore $\delta U$ corresponds to $0.3 \mathrm{mV}$, which gives, for a full range of $10 \mathrm{~V}$ an equivalent displacement $\Delta U=2 \times 10^{-5} \times 10 / 3 \times 10^{-4} L_{g}$. Consequently, the displacement range $\Delta U$ of the machine must be such that

$$
|\Delta U|<0.65 L_{g}
$$

In present case, the gauge length is $L_{g}=90 \mathrm{~mm}$ (Fig. 6), so that the displacement range is equal to $\pm 59 \mathrm{~mm}$ for a $\pm 10 \mathrm{~V}$ range. A displacement range of $\pm 12.5 \mathrm{~mm}$ is sufficient for the tests reported herein and therefore inequation (4) is satisfied. The testing machine gain $G_{1}$ is equal to $1.25 \mathrm{~mm} / \mathrm{V}$.

One of the most straightforward steps to tune such an integral controller is to model the system using Z-transform and then to proceed on by utilizing a computer-aided software to find the best parameters that meet the required performances such as stability, zero steadystate error and high dynamic response. However, since this work does not involve high control performances, and given the very slow dynamic response of the outer loop (owing to the very low sampling frequency compared with the high dynamic response of the inner loop), Laplace transforms are used to investigate the control scheme. It is worth remembering that Laplace transform theory is clearly unsuited to meet performance criteria (3). Since stability that is met as a first step is largely over-estimated, it makes no difference using Laplace transforms rather than Z-transforms.

If the machine PID controller (inner loop) is set in such a way that there is no overshoot, then the transfer function of the testing machine in a displacement-controlled mode is modeled as a first order system $G_{1} /\left(1+\tau_{m} s\right)$, with $\tau_{m} \approx 20 \mathrm{~ms}$ (Fig. 2b) for the sake of simplicity. This is possible since, with the above tuned condition, the system shows a dominant pole (i.e., a gain attenuation of about $-20 \mathrm{~dB} /$ decade after the cut-off frequency). However, since the system actual order is greater than one, the phase lag exceeds $-90^{\circ}$. The 
latter is considered in the phase margin calculations. There is an additional transfer function of gain $G_{0}=1 / L_{g}$ to drive the sample in a strain-controlled mode. The camera takes pictures at a maximum frame rate of 12 frames per second. Therefore, it delivers the frame with a time lag $\tau_{0}$ equal to $83 \mathrm{~ms}$. The optical sensor is an ideal low-pass filter with a cut-off frequency of $12 \mathrm{~Hz}$ and with a time lag $\tau_{0}$. This transfer function is not considered in the following analysis since its cut-off frequency is two orders of magnitude greater than that of the overall compensated system (i.e., $0.15 \mathrm{~Hz}$ ). Furthermore, as mentioned earlier, with the implemented DIC algorithm, the system sampling rate obtained is at most about $0.03 \mathrm{~Hz}$ (i.e., $\tau_{\mathrm{s}} \approx 6 \mathrm{~s}$ ). A zero-order hold is put in the loop to account for the sampling effects. Its transfer function reads $H_{0}(s)=H_{0} \exp \left(-\tau_{s} s / 2\right)$ where $H_{0}$ is a real number such that $\left|K_{0}\right| \leq 1$. In the following, the upper bound $\left|K_{0}\right|=1$ is considered since it leads to a lower bound to the phase margin. The integral controller by itself is modeled as having the following transfer function $K_{i} /$ s. Thus the open-loop transfer function of the overall system is given by $H_{0} K_{\mathrm{i}} G_{0} G_{1} \exp \left(-\tau_{s} s / 2\right) / s\left(1+\tau_{m} s\right)$. Figure 3a shows the latter gain as a function of the frequency and that of the inner loop (i.e., testing machine in a displacement-controlled mode). If the open loop constant gain is set to one, i.e., $K_{\mathrm{i}} G_{0} G_{1}=1$ (Fig. 3a), then the closed-loop system is stable and even over-damped with a phase margin of about $75^{\circ}$ (Fig. $3 \mathrm{~b}$ ). The phase lag induced by the sampling rate is also drawn on the same plot and was considered when computing the phase of the overall system. Consequently, the gain of the integral controller $K_{i}$ is equal to $126 \mathrm{~V}$.

This approach allows us to get a first guess of the controller integral gain that may be optimized afterwards by a trial and error method. Setting the open-loop gain to 1 in the openloop transfer function yields a slow system response that meets the stability criterion. Zero steady-state error is achieved by the integral feedback feature of the controller. The system performance is certainly not optimal but proved to be sufficient for the present application. 
However, if better properties are sought, a conventional PID controller should be implemented using special CAD softwares.

Figure 4 shows a flowchart of the algorithm. For security reasons, it is desirable to avoid strain steps exceeding $2 \times 10^{-4}$. Therefore, the feedback absolute error is clipped to $14 \mathrm{mV}$ with the present parameters. The DAC incremental steps are confined within a \pm 14 $\mathrm{mV}$ range with $K_{i}=126 \mathrm{~V}$. The integral feedback difference equation is

$$
u(k)=u(k-1)+\operatorname{clip}\left(T K_{i} \varpi(k)\right),
$$

where $u($.$) is the controller output, k$ the considered step, clip(.) the $14 \mathrm{mV}$-clipping function, $T$ the sampling period, $K_{i}$ the integral gain and $\varpi($.$) the error signal.$

CORRELI $^{\text {CONTROL }}$ was tested with the above value for $K_{i}$. The feedback control performances are examined by studying the system step response (Fig. 5). A careful look at the results shows that sixteen measurement points are needed for an approximate time lapse of 100 s, i.e., the sampling period is around 6 s. Furthermore, the strain error fluctuations at the controller output are less than those after the DIC calculations. This is explained by the fact that the controller performs subsequently an integration (Fig. 2b), which is known to filter noise. This result shows the robustness of the controller against strain fluctuations induced by DIC. Last, the feedback control step response is approached by that of a first order system with a time constant $\tau$ of $75 \mathrm{~s}$. Consequently, no overshoot occurs, which is beneficial to avoid sample failure, and the phase margin is of the order of $90^{\circ}$, in agreement with the $a$ priori analysis (Fig. 3b). CORRELI ${ }^{\text {CONTROL }}$ is now characterized and is used to perform an experiment.

\section{Tensile test with CORRELI ${ }^{\text {CONTROL }}$}

A tensile test is carried with CORRELI ${ }^{\text {CONTROL }}$ for a steel that is supposed to behave like an elastic perfectly plastic body. The sample is a coupon of size $157 \times 50 \times 2 \mathrm{~mm}^{3}$ 
prepared by spraying black/white paint to get a random texture suitable for DIC. A multiaxial testing machine (ASTRÉE) was chosen. In the present case, only two jacks associated with a single loading direction were used. ${ }^{3}$ In the future, it is planed to use the same system to control multiaxial experiments. A strain rosette was installed on the lower part of the specimen. As mentioned earlier, $3 \times 3$ ZOIs $\left(L_{\mathrm{ZOI}}=64\right.$ pixels $)$ are selected in the $550 \times 1000$-pixel ROI to control the experiment (Fig. 6). The minimum gauge length $L_{0}$ is equal to 400 pixels.

The strain history consists of successive loading/unloading steps up to $0.08 \%, 0.26 \%$, $0.48 \%, 0.9 \%$ and $1.5 \%$, respectively. Every loading step is followed by a corresponding unloading down to a force of about $7.5 \mathrm{kN}$. Given the relative slowness of the sampling rate due to image processing, the strain rate was left uncontrolled. Figure 7 shows the corresponding stress / strain curves for the strain rosette and CORRELI ${ }^{\mathrm{CONTROL}}$. At an early stage, the rosette delivers no meaningful results. Had it been used to control the experiment, it would have led to sample failure. However, it allows for the identification of the Young's modulus (i.e., $203 \pm 2 \mathrm{GPa}$ ), the Poisson's ratio (i.e., $0.27 \pm 0.01$ ), and yield stress (i.e., $275 \pm$ $5 \mathrm{MPa}$ ) of the material by using strain data and the applied load.

The data obtained by DIC are in good agreement with those given by the rosettes (Fig. 7). This validates the system in the elastic domain. To quantify strain uncertainties from these measurements, it is interesting to plot the difference between the first loading curves and their respective linear regressions. Figure 8 shows the noise signals defined as residues from a linear regression of the strain signals. The ratio between the RMS values of CORRELI ${ }^{\text {CONTROL }}$ noise to that of the strain gauge (noise) is about 2.3. The strain uncertainty obtained with CORRELI ${ }^{\text {CONTROL }}$ is of the same order of magnitude as that of the strain rosette. Better signal to noise ratio (SNR) is to be expected from a camera whose dynamic range is 12 bits or over. $^{3}$ Furthermore, the standard strain uncertainty $\rho_{\varepsilon}$ is of the order of 
$1.1 \times 10^{-5}$ in agreement with the a priori uncertainty analysis performed in Section 2 . This value is less than those observed with artificial or natural random patterns ${ }^{3,7,13}$ with 8 -bit pictures. The main difference is due to the dynamic range of the CCD sensor (here equal to 10.5 bits) and the gauge length.

It was observed that the strains recorded by the rosettes in the elasto-plastic region do not follow those given by CORRELI ${ }^{\text {CONTROL }}$ (Fig. 7). A full-field displacement analysis with the images acquired by CORRELI ${ }^{\text {CONTROL }}$ is necessary and discussed now. Figure 9 shows displacement fields given by a classical DIC software, here CORRELI ${ }^{\mathrm{LMT}}{ }^{4}$ To get a better spatial description, the ZOI size is less than that of CORRELI ${ }^{\text {CONTROL }}$, namely, $L_{Z O I}=32$ pixels and the distance $\delta$ between two neighboring measurement points is equal to 32 pixels. From Fig. 1, it is expected that the displacement uncertainty be not as low as that of CORRELI ${ }^{\mathrm{CONTROL}}$. In the elastic domain, the displacement field corresponds to that of a homogenous tensile test. Conversely, shear bands appear in the lower and upper right corners of the ROI in the elasto-plastic region (Fig. 9). The rosette (Fig. 6) is thus traversed by the lower shear band. From the rosette signal, it is assumed that one part of it is coming off so that the plastic strains cannot be used. This shows another advantage of CORRELI ${ }^{\text {CONTROL }}$. It allows for a later DIC analysis to get the displacement and strain fields in the region of interest. Strain gauges or extensometers cannot give that type of information. Furthermore, had the experiment been controlled by using the strain rosette, the macroscopic plastic strains could not have been controlled and the experiment would have been of no use. Last, the strain values obtained with CORRELI ${ }^{\text {CONTROL }}$ are in very good agreement with average values obtained with CORRELI ${ }^{\mathrm{LMT}}$. This validates the system in the plastic regime.

\section{Conclusion}

It was shown that digital image correlation can be used to control a mechanical test. An ad hoc software (CORRELI ${ }^{\mathrm{CONTROL}}$ ) was developed to deal with particular aspects. 
Because of heavy image processing and relatively lengthy image storage, the overall working frequency is about $0.15 \mathrm{~Hz}$. This low cycle time led us to implement a two-loop-cascade control scheme. An inner quick (wired) displacement-controlled loop is driven by a slow outer strain-controlled loop. The latter was devised with the specification of a zero steady state error condition. Consequently, only quasi-static tests were performed up to now. It is envisioned that a real-time system is feasible when the pictures are not stored and computations optimized. The software responded in a correct way and proved to be successful in driving an experiment on a steel coupon in the elastic and plastic regions.

CORRELI $^{\text {CONTROL }}$ needs very few tuning, and promising results were obtained from actual tests. A pragmatic approach was followed to determine the gains by avoiding a lengthy identification process. Furthermore, the specification related to the security of the experiment (i.e., avoiding premature failure induced by the control system) was of paramount importance compared with its fast response, which is usually one key criterion. Therefore, it is believed that the system can still be optimized by relaxing the clipping level chosen herein. This will depend on the type of behavior that is investigated.

With the use of a 10.5-bit CCD camera, a standard displacement uncertainty of $5 \times 10^{-3}$ pixel and a standard strain uncertainty of the order of $10^{-5}$ are achieved so that the two-loop system has performances suited to most material behaviors, even in their elastic domain. In the present case, the strain uncertainty of DIC is only two times greater than that of strain gauges, both used in a standard way compatible with a mechanical test.

Pictures of the loaded specimen were saved during the test. One of the advantages of digital image correlation compared with strain gauge measurements was highlighted when reprocessed. By using a full-field measurement by digital image correlation software, it is concluded that shear bands appear on the specimen surface in the elasto-plastic region. Strain gauges could not record this phenomenon. 


\section{Acknowledgements}

The authors express their gratitude to Dr. Yvan Bonnassieux for useful and stimulating

discussions on control issues. The authors thank the anonymous reviewers for their helpful suggestions. 


\section{References}

1. Lagarde, A. (ed.) Advanced Optical Methods and Applications in Solid Mechanics (Kluwer, Dordrecht (the Netherlands), 2000).

2. Rastogi, P. K. (ed.) Photomechanics (Springer, Berlin (Germany), 2000).

3. Périé, J. N., Calloch, S., Cluzel, C. \& Hild, F., "Analysis of a Multiaxial Test on a C/C Composite by Using Digital Image Correlation and a Damage Model," Exp. Mech., 42, 318328 (2002).

4. Hild, F., Raka, B., Baudequin, M., Roux, S. \& Cantelaube, F., "Multi-Scale Displacement Field Measurements of Compressed Mineral Wool Samples by Digital Image Correlation," Appl. Optics, IP 41, 6815-6828 (2002).

5. PCO, "PixelFly High Performance Digital CCD Camera System," http://www.pco.de, (2000).

6. ISO, "International Vocabulary of Basic and General Terms in Metrology (VIM)," International Organization for Standardization, Geneva (Switzerland), (1993).

7. Bergonnier, S., Hild, F. \& Roux, S., "Strain heterogeneities in tension and compression tests on mineral wool samples," J. Strain Analysis, 40, 185-197 (2005).

8. Press, W. H., Teukolsky, S. A., Vetterling, W. T. \& Flannery, B. P., "Numerical Recipes in Fortran," Cambridge University Press, Cambridge (USA), (1992).

9. ISO, "Guide to the Expression of Uncertainty in Measurements (GUM)," International Organization for Standardization, Geneva (Switzerland), (1995).

10. Franklin, G. F., Powell, J. D. \& Emami-Naeini, A., "Feedback control of dynamic systems, third edition," Addison-Wesley, Reading, MA (USA), (1994).

11. Rivoire, M. \& Ferrier, J.-L., "Cours d'automatique. Commande par calculateuridentification," Eyrolles, Paris (France), (1990). 
12. National Instrument, "LabVIEW $W^{T M}$ version 5.1, user's manual (http://www.ni.com)," (1999).

13. Forquin, P., Rota, L., Charles, Y. \& Hild, F., "A Method to Determine the Toughness Scatter of Brittle Materials," Int. J. Fract., 125, 171-187 (2004). 


\section{List of figures}

Figure 1: Standard displacement and strain uncertainties as functions of ZOI size. The solid symbols are correlation results. The solid line is the best fit by a power law of exponent -1.5 , and the dashed line is the best fit by a power law of exponent -2.5. In the present case, the strain gauge length is equal to twice the ZOI size.

Figure 2: -a-Schematic of a cascade feedback using digital image correlation to control an electro-hydraulic testing machine. -b-Block diagram of the cascade feedback. Points A and B correspond to the signals shown in Fig. 5.

Figure 3: Bode plot of the overall system and the inner loop (testing machine in displacement controlled mode). -a- Gain variation with frequency. -b- Phase vs. frequency. The phase lag induced by the sampling rate is also plotted.

Figure 4: Flowchart of the cascade feedback.

Figure 5: Step response of the system and first order description with a time constant $\tau=75 \mathrm{~s}$. The latter was determined from the fit by a first order system. Points A and B are depicted in Fig. 2.

Figure 6: Sample geometry, location of the strain rosette, and ZOIs within the chosen ROI.

Figure 7: Stress vs. longitudinal and transverse strains obtained by using strain rosette and digital image correlation data (about $2 \times 1000$ measurement points are shown).

Figure 8: Linearity gap for different measurement points for a strain rosette and digital image correlation in the elastic domain. The horizontal lines correspond to the standard uncertainty for the strain rosette and digital image correlation. 
Figure 9: Displacement fields (magnification: $\times 38$ ) determined by digital image correlation $\left(L_{Z O I}=32\right.$ pixels, $\delta=32$ pixels $)$ for different longitudinal strain levels of a tensile test. 


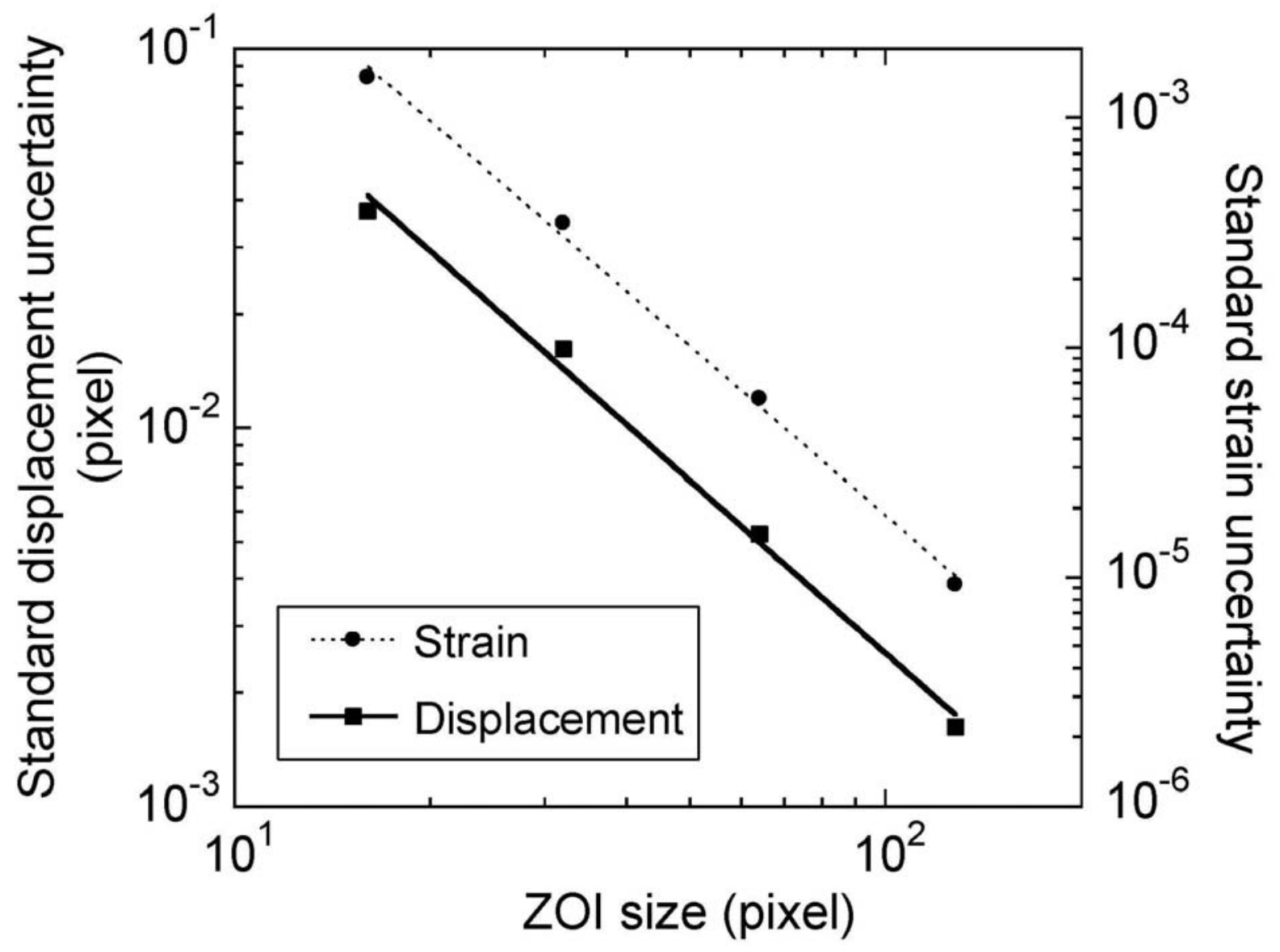

Figure 1: Fayolle et al. 


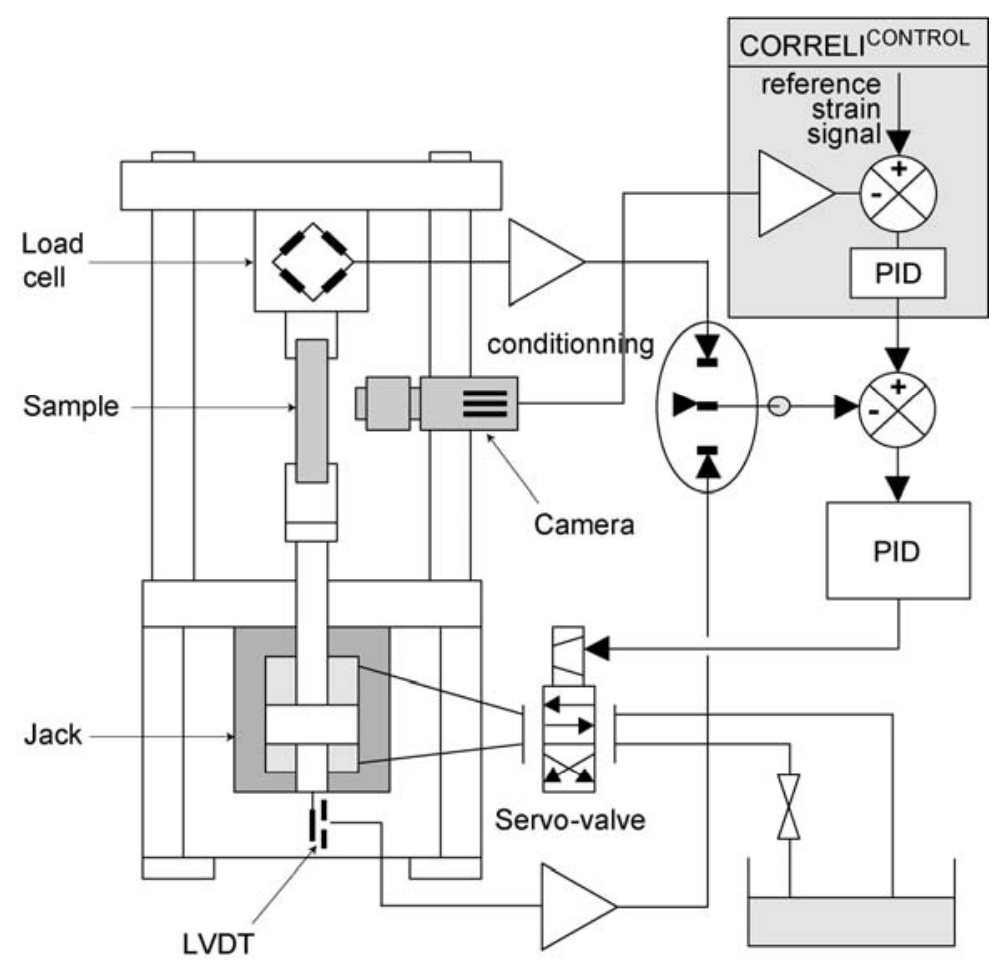

$-a-$

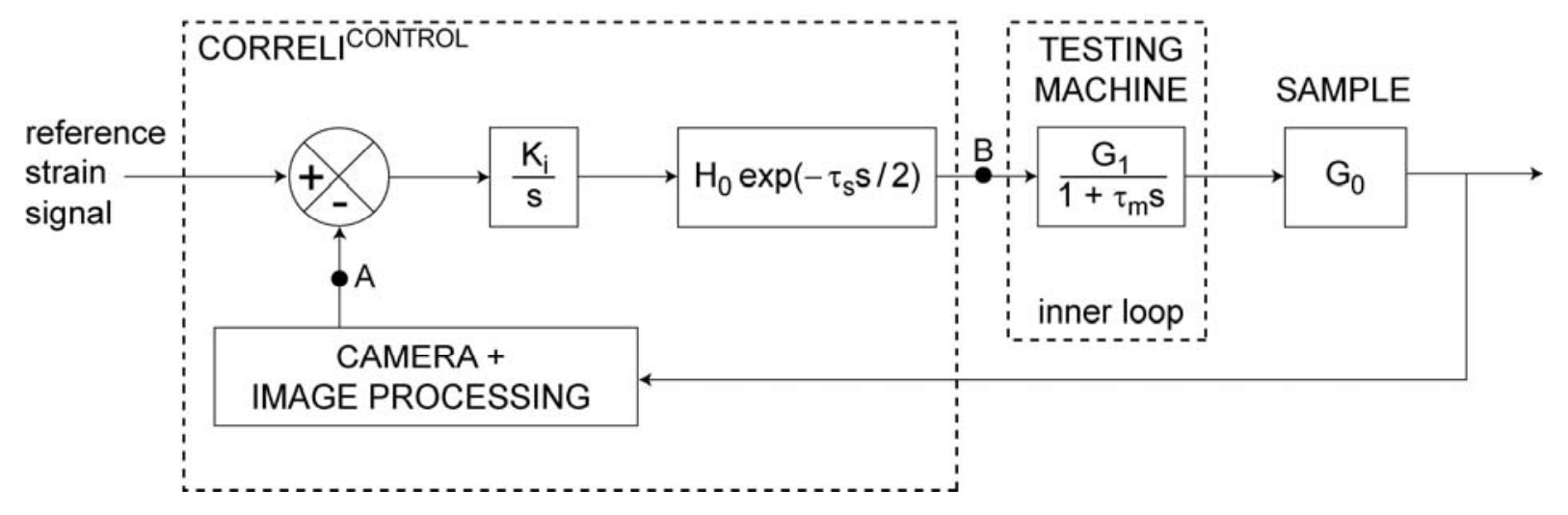

-b-

Figure 2: Fayolle et al. 


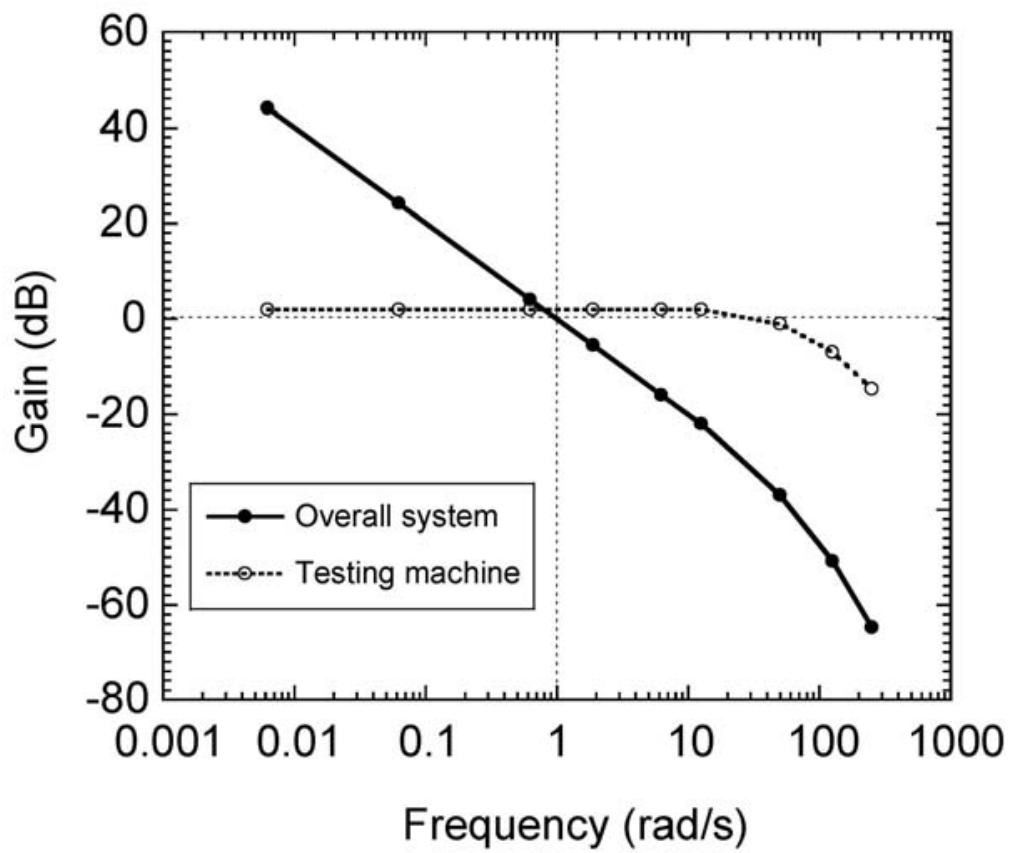

$-a-$

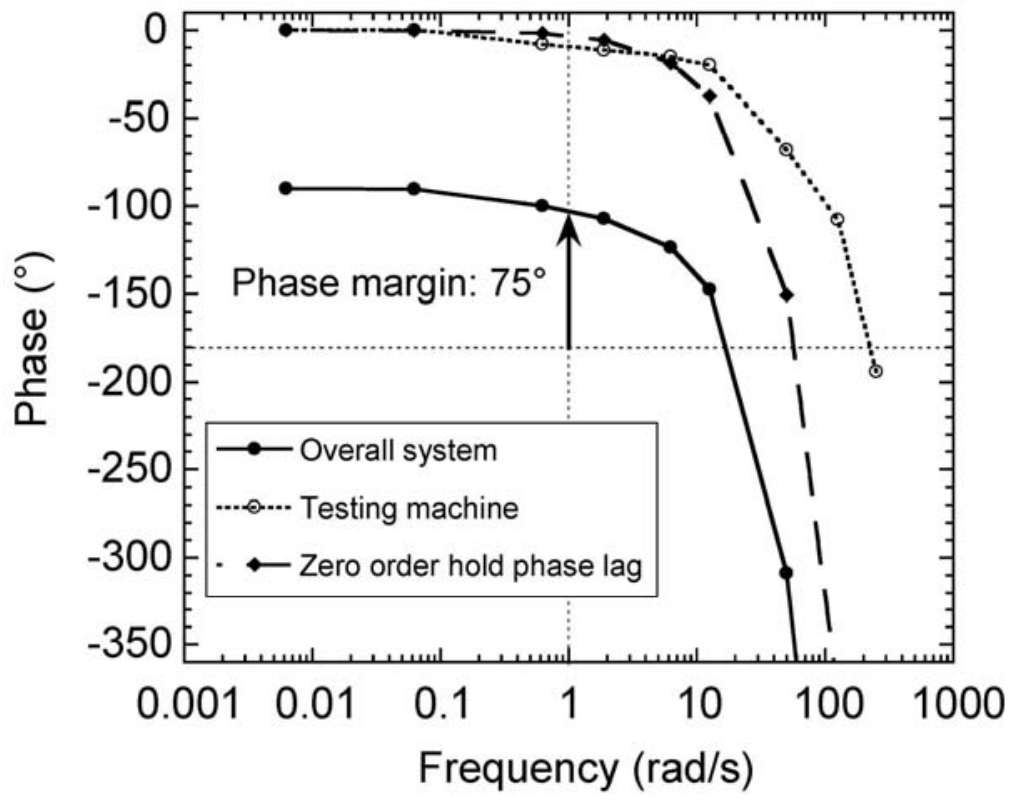

-b-

Figure 3: Fayolle et al. 


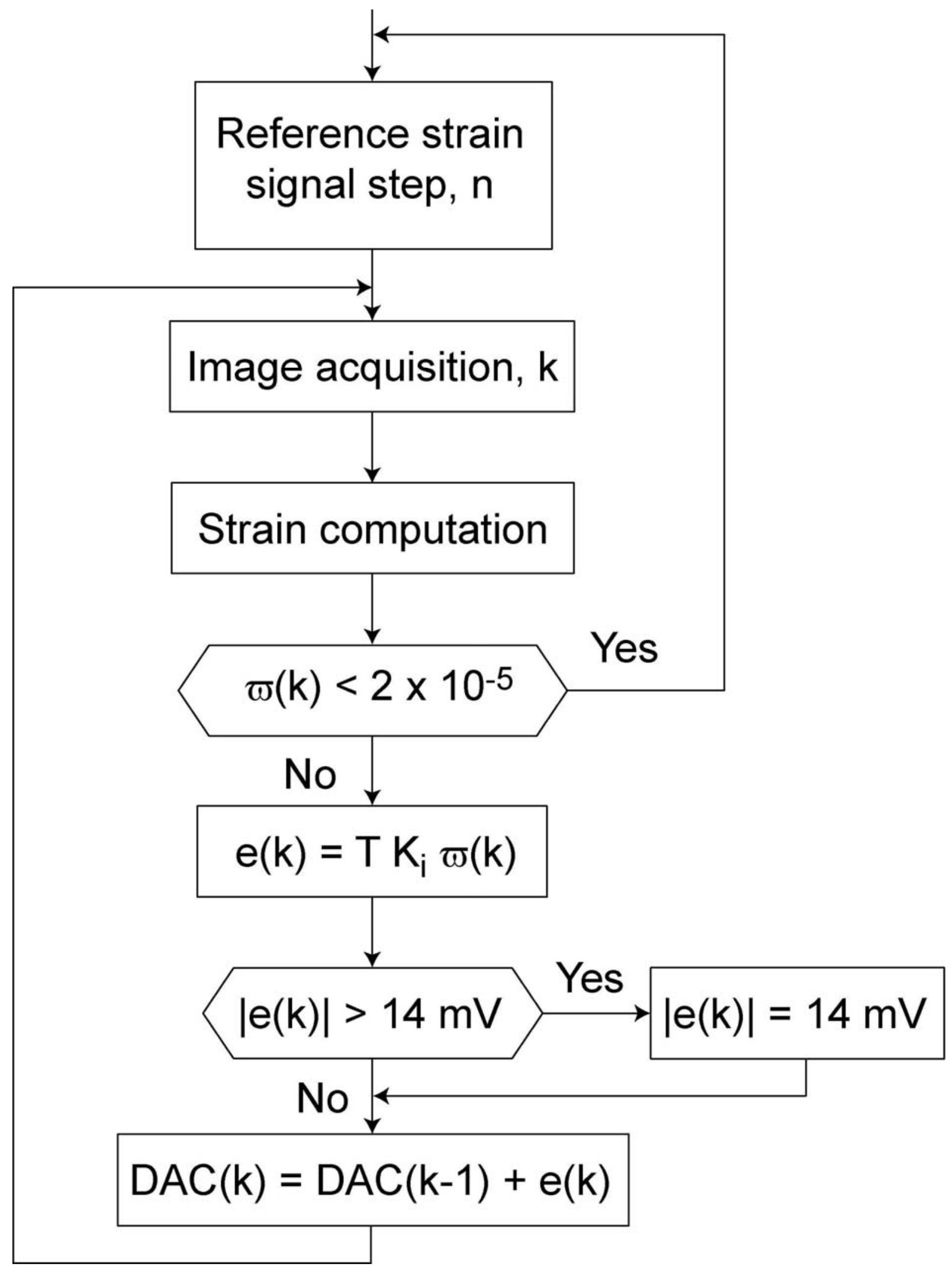

Figure 4: Fayolle et al. 


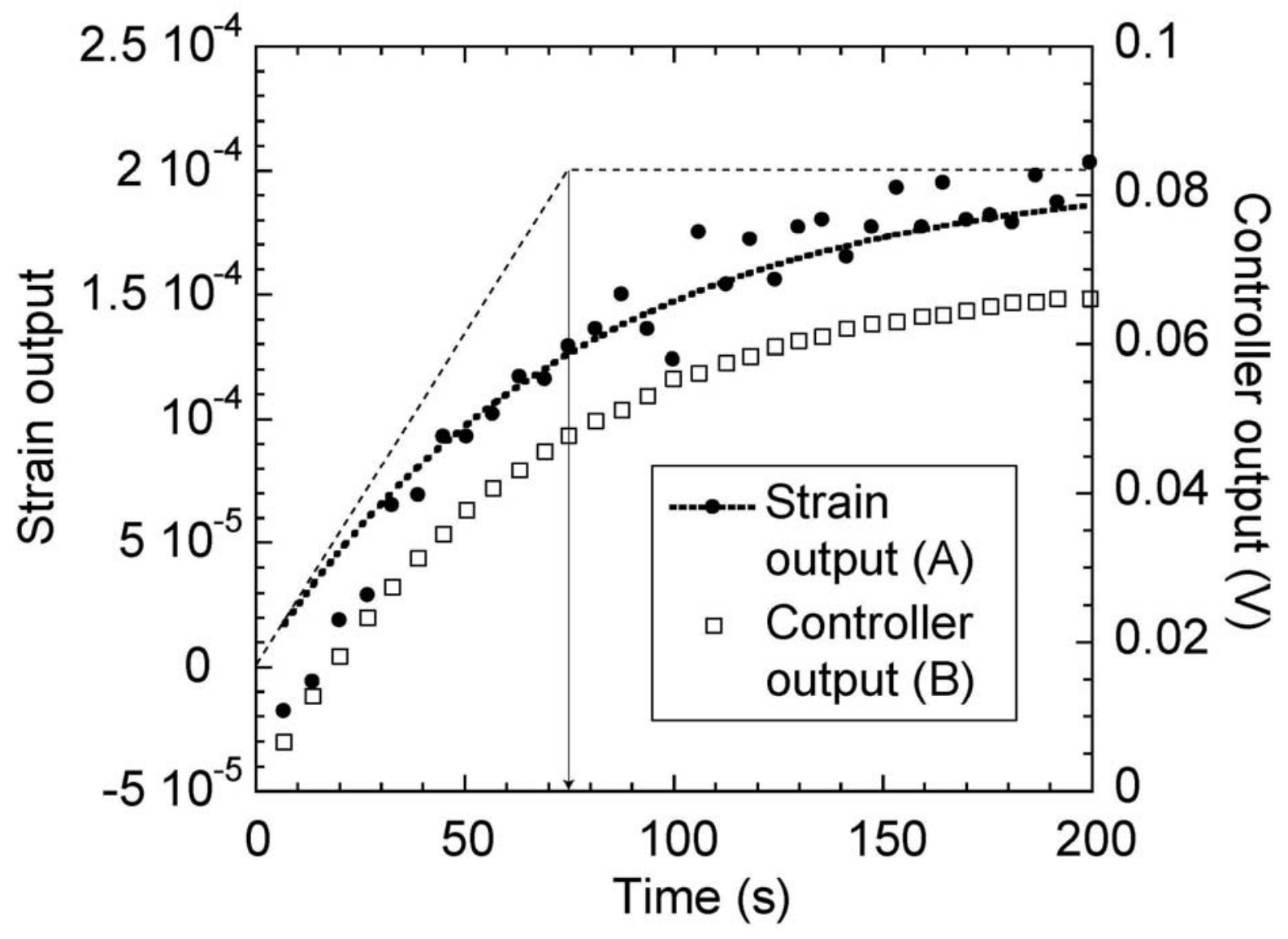

Figure 5: Fayolle et al. 


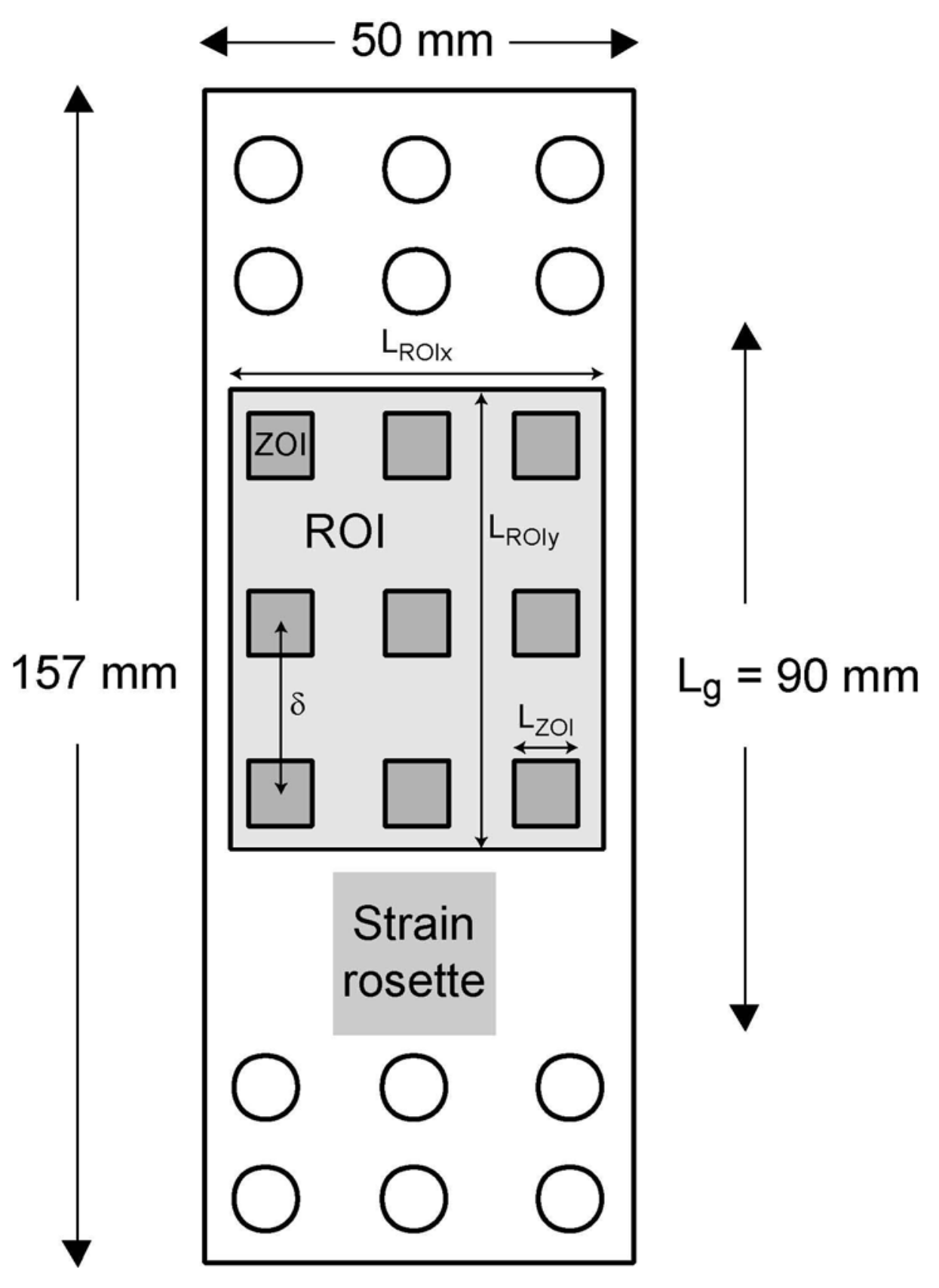

Figure 6: Fayolle et al. 


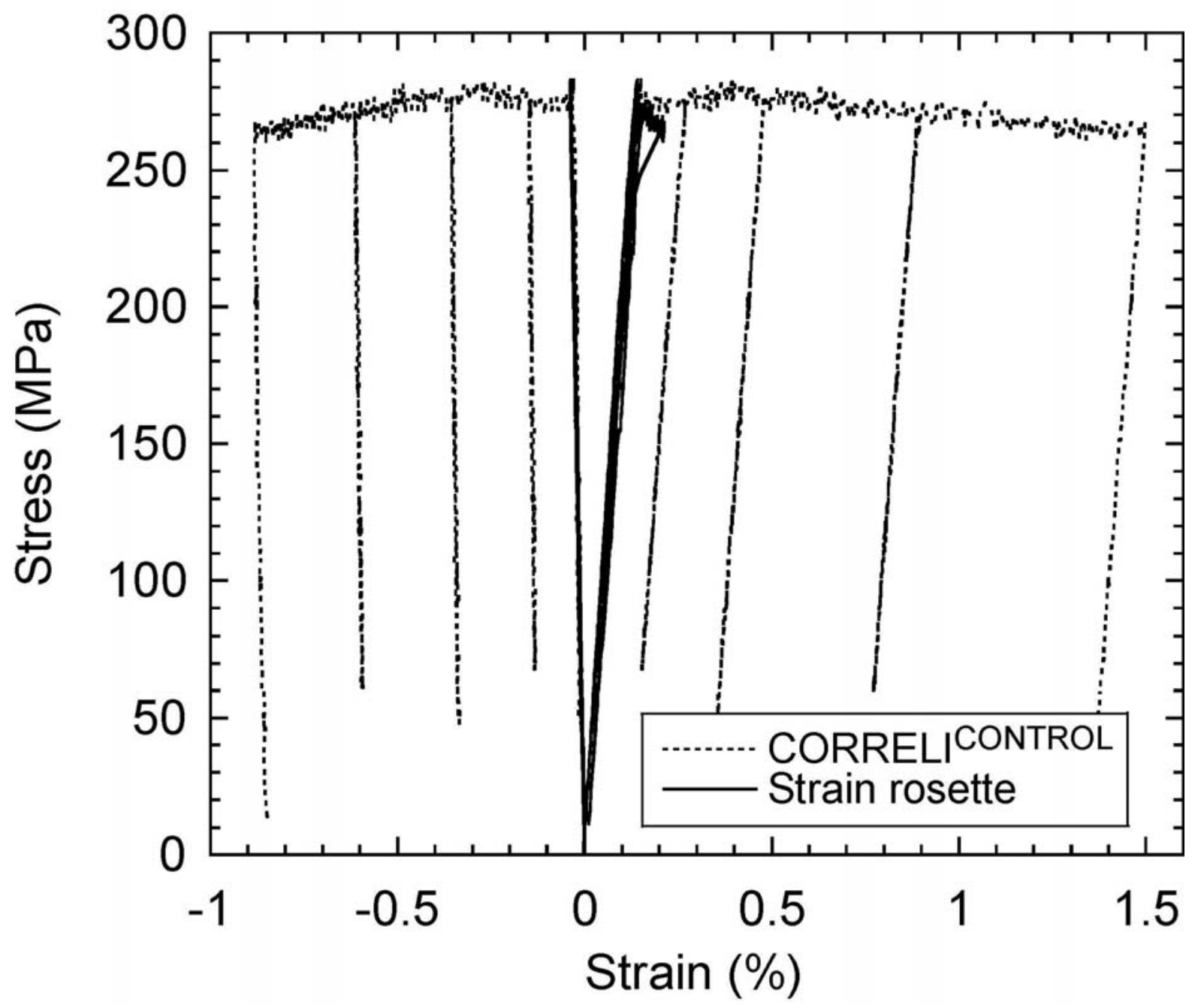

Figure 7: Fayolle et al. 


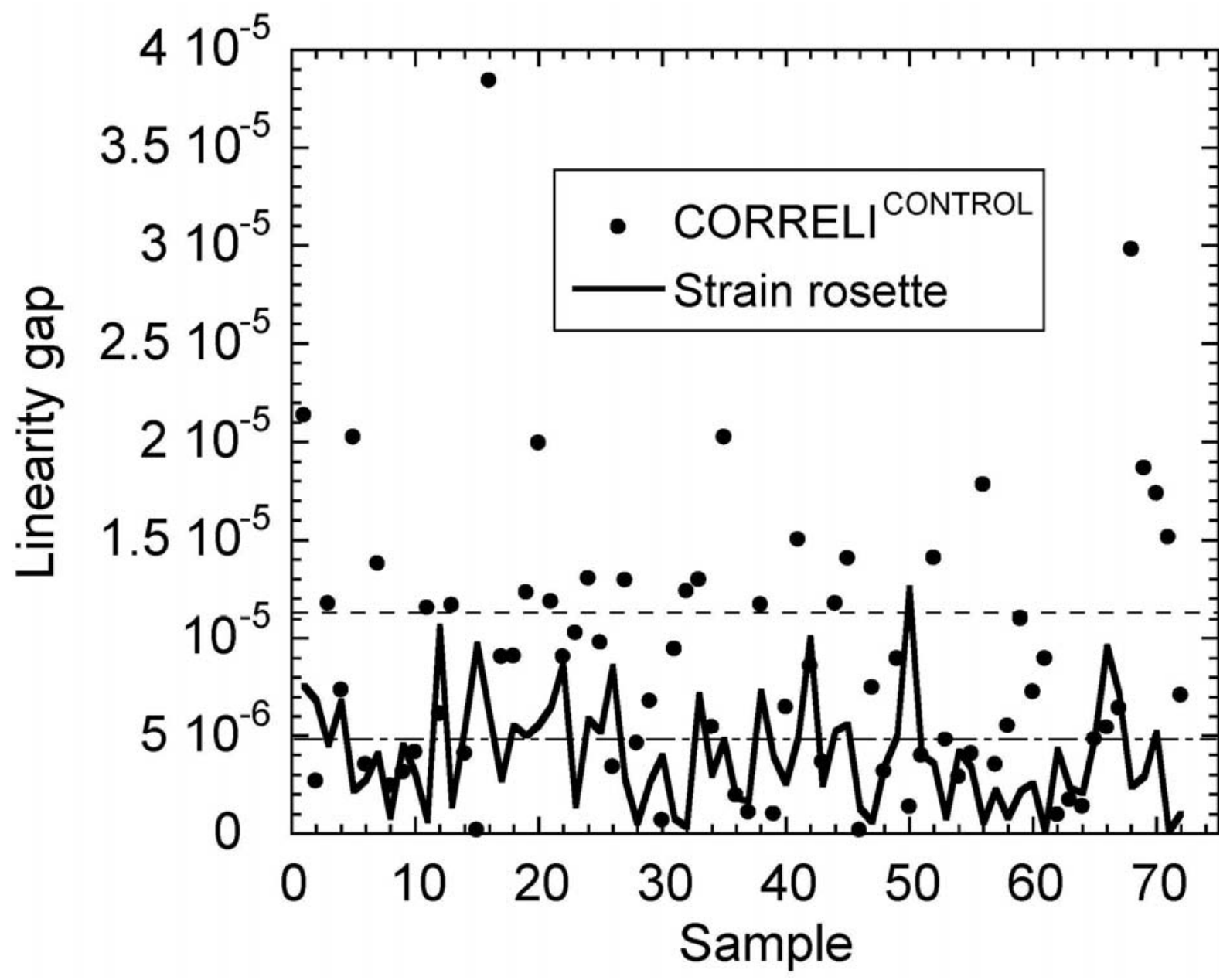

Figure 8: Fayolle et al. 


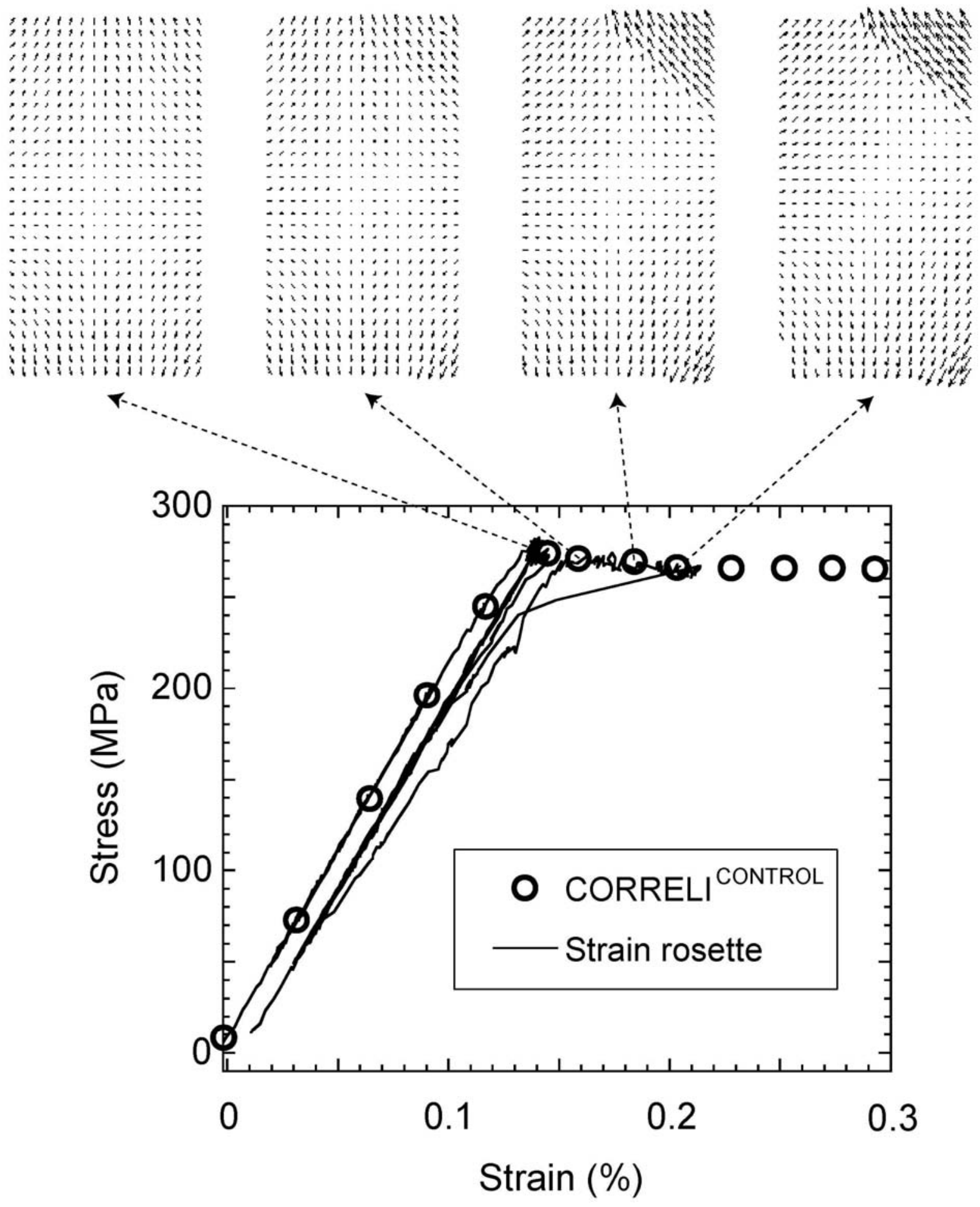

Figure 9: Fayolle et al. 